Layered Oxide Cathodes for Li-Ion Batteries: Oxygen Loss and Vacancy

\title{
Evolution
}

Hanlei Zhang1, 2, Brian M. May2, 3, Fredrick Omenya², M. Stanley Whittingham², Jordi Cabana ${ }^{2,3}$ and Guangwen Zhou $^{1,2 *}$

1. Materials Science and Engineering Program \& Department of Mechanical Engineering, State University of New York, Binghamton, New York 13902, United States

2. NorthEast Center for Chemical Energy Storage, State University of New York, Binghamton, New York 13902, United States

3. Department of Chemistry, University of Illinois at Chicago, Chicago, Illinois 60607, United States

Table of supplementary content

Section I: Description of the Supplementary Movie

Section II: Analysis of Oxygen Vacancies in the Layered Phase

Section III: Bulk Morphology at Stage 2

Section IV: EDS Analysis the Amorphized Rock-Salt Phase

Section V: Repeated Observations of the Preferred Formation of the RockSalt Phase along the Lithium Channels

Section VI: Surface of the 30-Cycle TODA-NCA

Section VII: Electrochemistry of TODA-NCA 


\section{Section I: Description of the Supplementary Movie}

Supplementary Movie_1. In-situ TEM observation of an NCA particle being held at $400{ }^{\circ} \mathrm{C}$ in the reducing environment generated by the high vacuum in the TEM chamber. The frame speed of the movie is $32 x$ real time.

Supplementary Movie_2. In-situ TEM imaging and FFT of the layered phase showed no phase transformation in the surface region after $\sim 30$ min of e-beam illumination of $\sim 200 \mathrm{e} / \AA^{2} \cdot \mathrm{s}$ at room temperature. The movie represents $32 \mathrm{~min} 53 \mathrm{~s}$ in real time, which is sped up by 64 times of the actual time. A video clip showing the relative position of Movie_2 is presented in Figure S1.

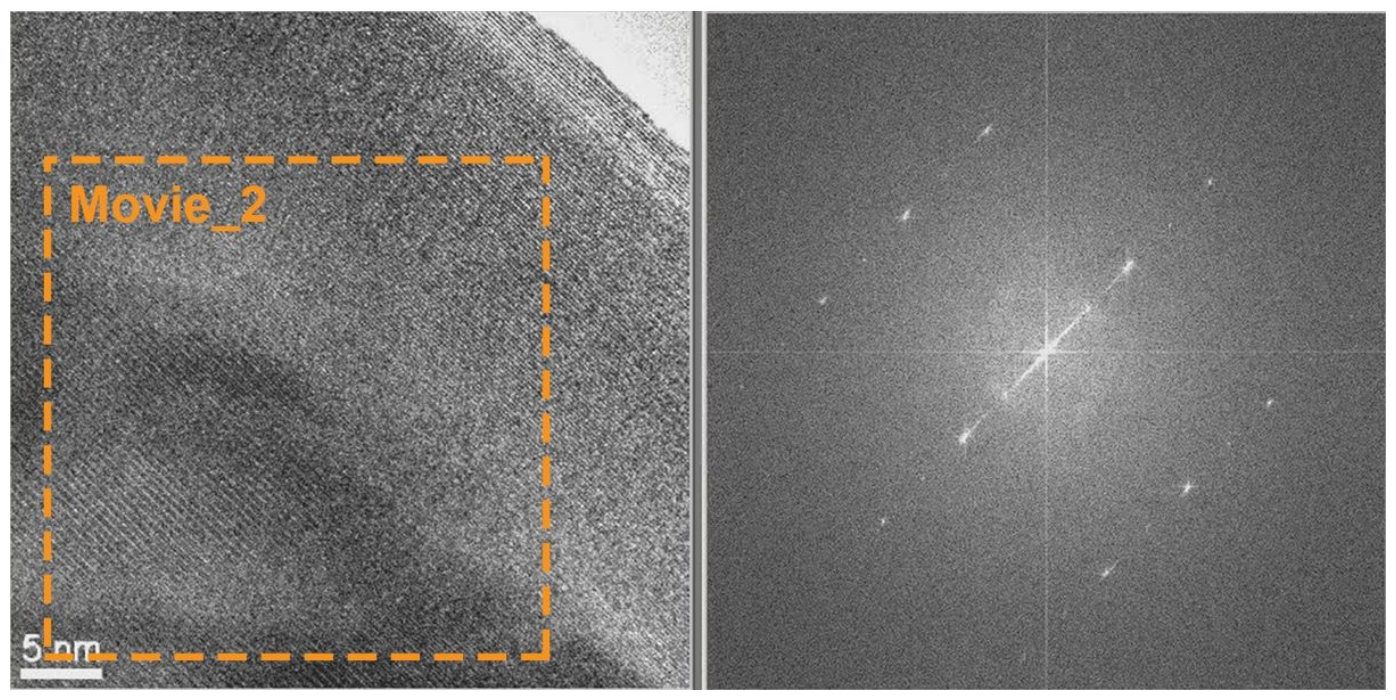

Figure S1 | Video Clip showing the area (dashed square) from which Movie_2 is captured. 


\section{Section II: Analysis of Oxygen Vacancies in the Layered Phase}

Figure S2 presents a magnified HRTEM view of the oxygen vacancies in the layered phase, which is evidenced by its dark contrast, as marked with a yellow arrow. The blue lines mark out the correspondence of TM cations in neighboring slabs, which indicates that the dark contract is not generated by missing a TM column, but via elongating the distance between neighboring TM cations. The distorted positioning of TM cations indicates that the oxygen frame work is defective, resulting from the adoption of oxygen vacancies.

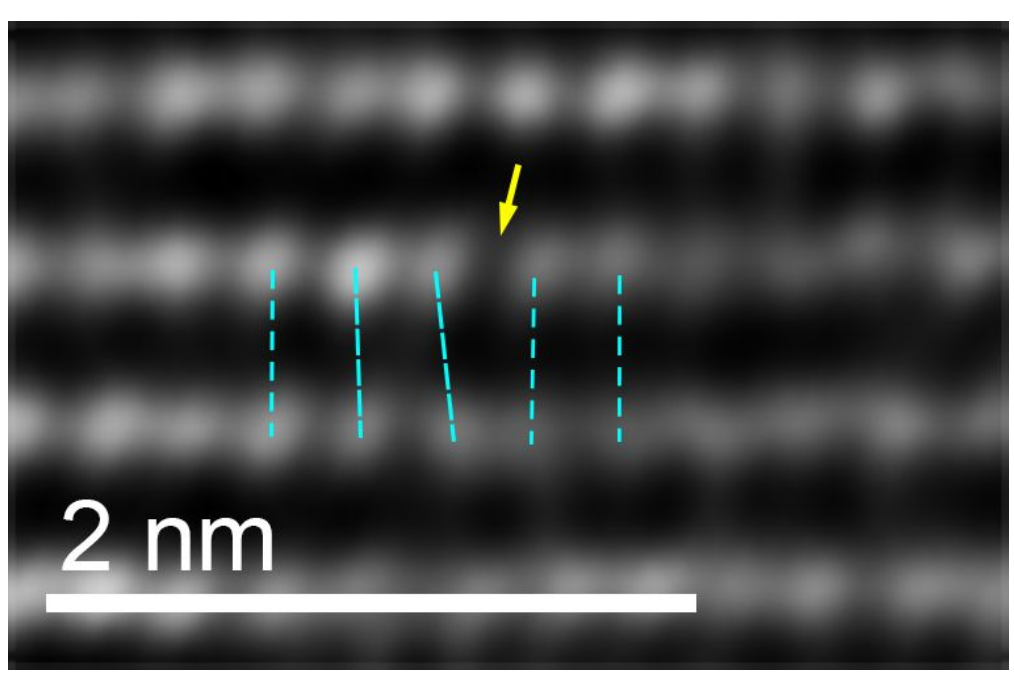

Figure S2 | Magnified HRTEM image of the oxygen vacancy in the layered phase, as marked with a yellow arrow. 


\section{Section III: Bulk Morphology at Stage 2}

Figure S3 presents an HRTEM image of the layered phase in the bulk at stage 2, which shows that oxygen vacancies are still present in the framework of the layered phase, comparable to that of stage 1 . The presence of oxygen vacancies can be evidenced by a wavy pattern of the atomic planes as well as the distorted positioning and weaken contrast of some atomic columns, as indicated by the yellow arrows.

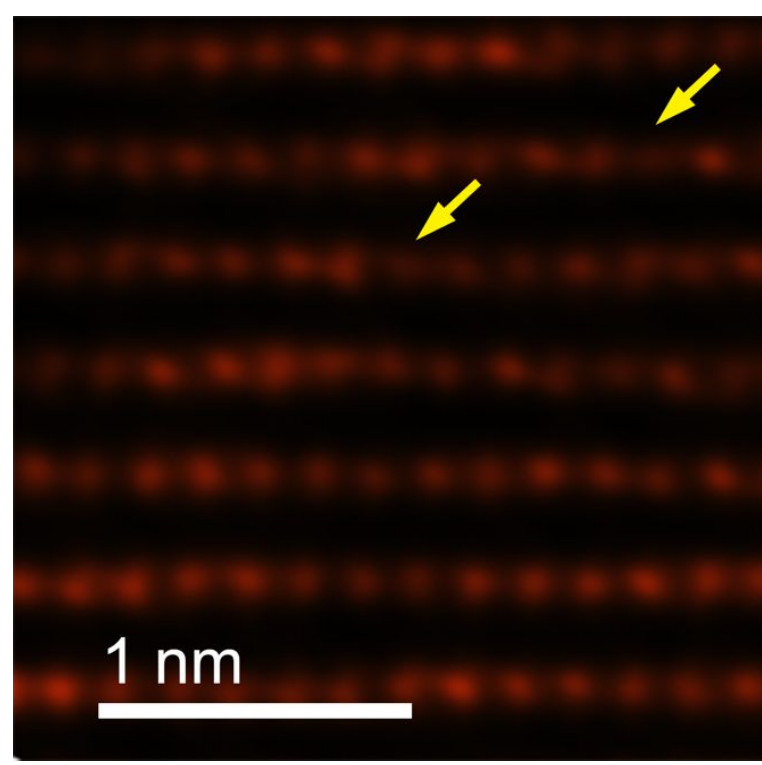

Figure S3 | HRTEM image of the layered phase with oxygen vacancies in the bulk at stage 2 , comparable to the layered phase at stage 1. 


\section{Section IV: EDS Analysis the Amorphized Rock-Salt Phase}

Figure S4(a) presents the STEM-HAADF image of a particle kept at $400{ }^{\circ} \mathrm{C}$ for 7 min with the formation of an amorphized surface rock-salt layer (the same particle in Figures $6(a, b))$. EDS spectra were obtained from the bulk towards the surface, at spots 1, 2 and 3 marked in Figure S4(a). The corresponding EDS spectra are shown in Figures S4(b-d), and their corresponding chemical compositions are presented in Table S1. As can be seen, the oxygen content decreases from the bulk towards the surface, confirming that the amorphized rock-salt phase is oxygen-deficient compared with the pristine layered phase.
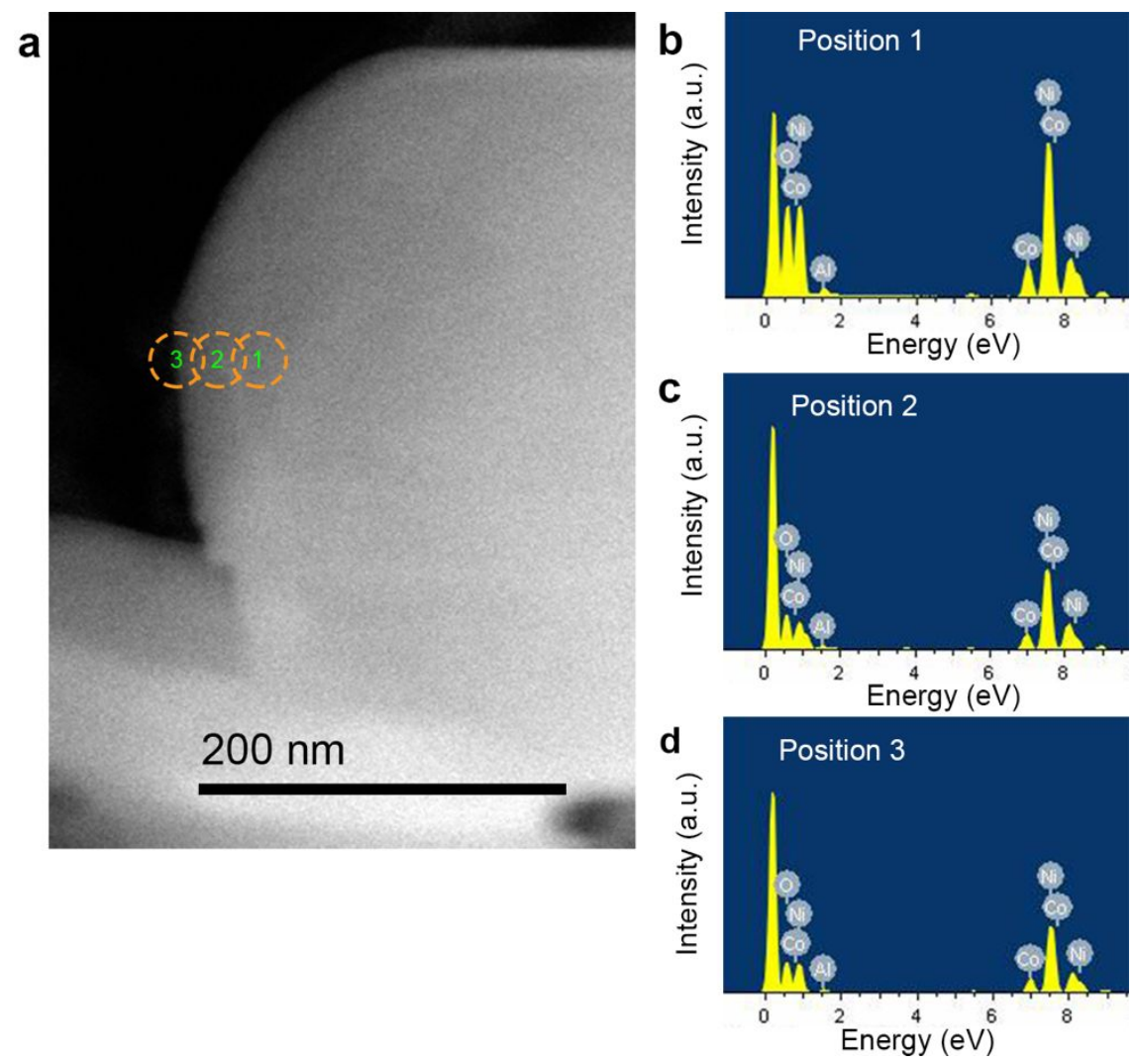

Figure S4 I EDS analysis of the amorphized rock-salt phase. (a) STEM-HAADF image of a particle with the amorphized rock-salt phase in the surface. (b-d) EDS spectra from positions 1-3, as marked in (a). 
Excluding lithium, the composition of oxygen in the layered phase is $66.67 \%$ and $50 \%$ in the rock-salt phase. All the values in Table S1 are between $50 \%$ and $66.67 \%$. Because the surface rock-salt layer is only a few nanometers thick, the e-beam always strikes on both the layered and rock-salt phases during the EDS analysis, yielding an arithmetic average of both.

Table S1 | Compositions of positions 1-3 in Figure S4.

\begin{tabular}{|c|c|c|c|c|}
\hline & O (atm. \%) & Ni (atm. \%) & Co (atm. \%) & Al (atm. \%) \\
\hline Position 1 & 62.87 & 29.88 & 5.70 & 1.54 \\
\hline Position 2 & 60.27 & 32.14 & 6.05 & 1.54 \\
\hline Position 3 & 59.35 & 32.76 & 6.43 & 1.45 \\
\hline
\end{tabular}


Section V: Repeated Observations of the Preferred Formation of the Rock-Salt

\section{Phase along the Lithium Channels}

Figure S5 presents HRTEM images of a particle kept at $400{ }^{\circ} \mathrm{C}$ for $7 \mathrm{~min}$, similar as the one in Figure 6(a). The rock-salt layer along the direction of the lithium channels is $\sim 15 \mathrm{~nm}$ while the one on the $(003)$ surface is $<1 \mathrm{~nm}$, confirming the preferred formation of the rock-slat layer along the direction of the lithium channels.
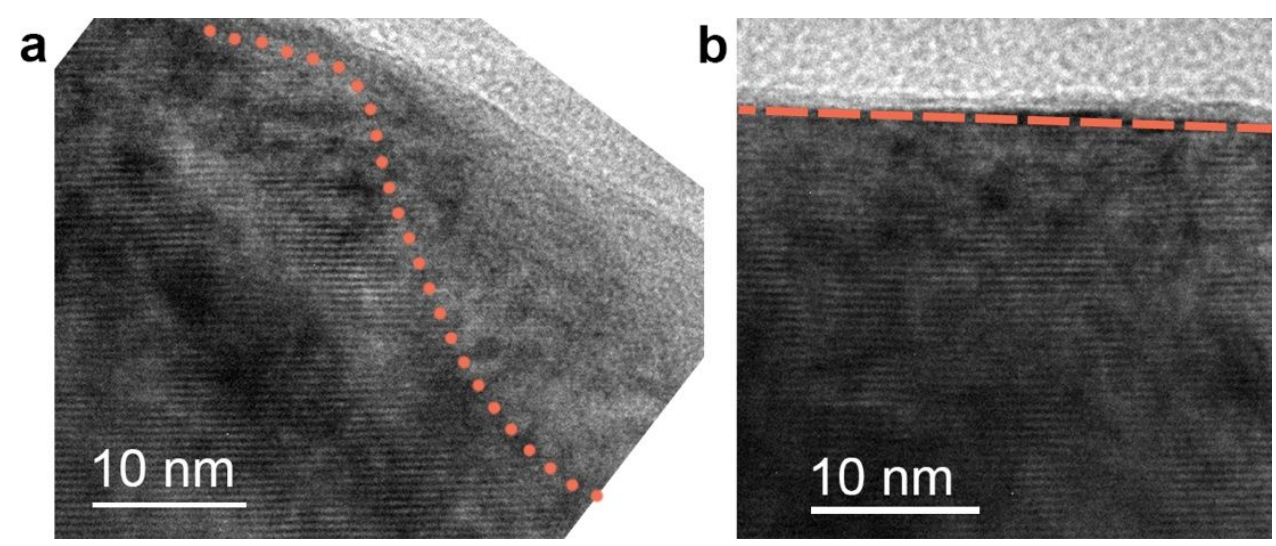

Figure S5 | Repeated observations of the preferred formation of the rock-salt phase along the lithium channels. $(a, b)$ HRTEM images of the surface rock-salt layers on an NCA particle kept at $400{ }^{\circ} \mathrm{C}$ for $7 \mathrm{~min}$. 
Section VI: Surface of the 30-Cycle TODA-NCA

Figure 66 presents an HRTEM image showing the surface region of the 30-cycle TODA-NCA cathode shown in Figures $7(a-d)$. As can be seen, the thickness of the surface-rock-salt layer is $\sim 5-6 \mathrm{~nm}$.

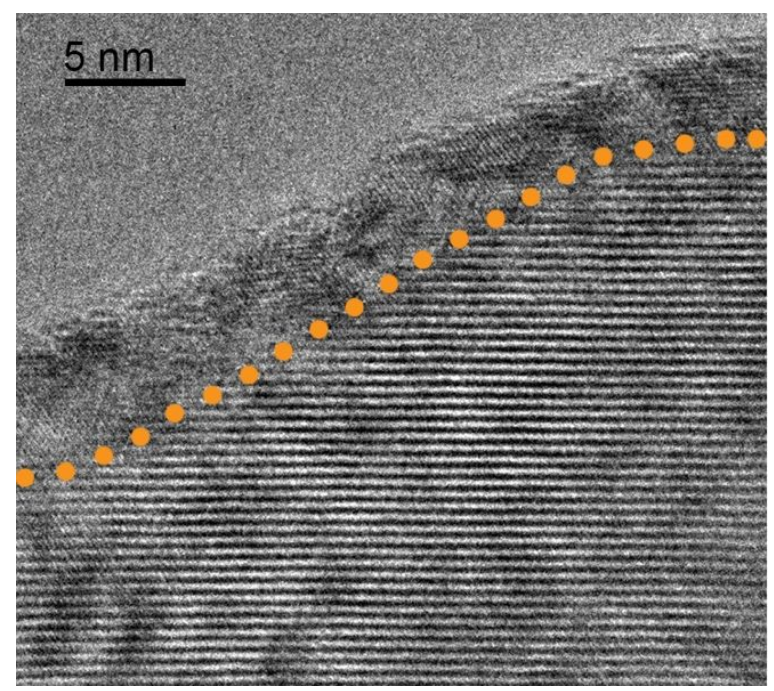

Figure S6 | HRTEM image showing the surface region of the 30-cycle TODA-NCA cathode. 


\section{Section VII: Electrochemistry of TODA-NCA}

Figure S7 confirms the abrupt capacity loss that occurs around the 50th cycle of the TODA-NCA cathodes cycled between $3.0-4.3 \mathrm{~V}$ at a rate of $\mathrm{C} / 10$. Figure $\mathrm{S} 7(\mathrm{a})$ presents two 300-cycle cells (the cell shown in Figure $7(\mathrm{f})$ is the cell 1 from Figure S7(a)). Figure S7(b) presents two 150-cycle cells, and Figure S7(c) presents a 100cycle cell. As can be seen, the abrupt capacity loss around the 50th cycle is observed in all the curves, confirming that it is an established phenomenon rather than a random artifact.
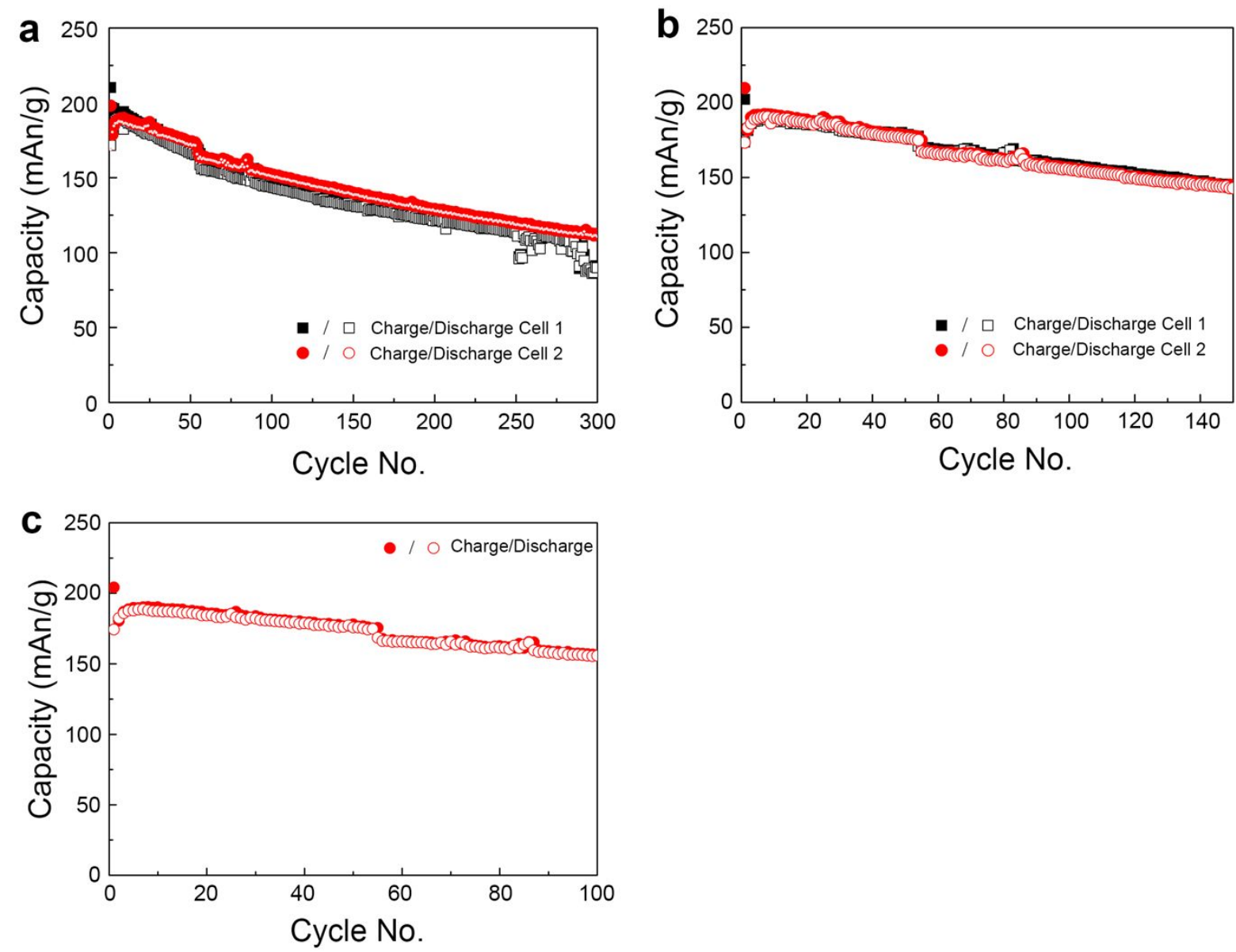

Figure S7 | Capacity vs cycle number curves of TODA-NCA. (a) Two 300-cycle cells. (b) Two 150-cycle cells. (c) A 100-cycle cell. 